\title{
Target versus non-target lesions in determining disease progression: analysis of 545 patients
}

\author{
S Raskin", E Klang, M Amitai \\ From International Cancer Imaging Society Meeting and 15th Annual Teaching Course (ICIS 2015) \\ London, UK. 5-7 October 2015
}

\begin{abstract}
Aim
RECIST and other methodologies emphasise tracking the diameters of target lesions (TLs) for the determination of progressive disease (PD) in randomised clinical trials. However, RECIST 1.1 also allows for the use of non TL lesions (NTL) to determine PD. We sought to determine whether the final assessment of PD was more likely to be determined by the set of target lesions with PD (TL-PD) or by the set of non-target lesions with PD (NTL-PD).
\end{abstract}

\section{Methods}

We evaluated the formal RECIST evaluations for consecutive patient enrollments in randomised clinical trials at our institution from 2012 to 2014. Data were grouped as TL-PD or NTL-PD, and the groups were compared. $\mathrm{PD}$ was further divided as to whether lesions were new or demonstrated unequivocal progression.

\section{Results}

Of 545 cases, 341 received a final assessment of PD. Of these, TL-PD analysis by itself accounted for 180 (53\%) patients, and NTL-PD analysis itself accounted for 287 (84\%). In the NTL-PD group, 142 (42\%) had either Partial Response (PR) or Stable Disease (SD) according to TL analysis. Among all instances of NTL-PD, new, measurable disease was the most common determinant of $\mathrm{PD}$, as seen in $210(73 \%)$ instances.

\section{Conclusion}

In this series of patients enrolled in clinical trials, NTL analysis was more likely to result in a determination of PD than tracking TL diameters, and the development of new, measurable disease was the most common determinant of PD. These findings may have relevance for

\footnotetext{
* Correspondence: stephen.raskin@sheba.health.gov.il
} Sheba Medical Center, Ramat Gan, Israel

c 2015 Raskin et al. This is an Open Access article distributed under the terms of the Creative Commons Attribution License (http:// creativecommons.org/licenses/by/4.0), which permits unrestricted use, distribution, and reproduction in any medium, provided the original work is properly cited. The Creative Commons Public Domain Dedication waiver (http://creativecommons.org/publicdomain/ zero/1.0/) applies to the data made available in this article, unless otherwise stated. 